\title{
Recent trends in interactive multimedia computing for industry
}

\author{
Raouf Boutaba · Kyung-Yong Chung · Mitsuo Gen
}

Published online: 15 February 2014

(C) Springer Science+Business Media New York 2014

As the development of IT convergence technology reaches its zenith, data in almost all areas have been developed and operated as knowledge based system after digitalization [1]. Interactive multimedia computing services allow people to access rich multimedia content anytime and anywhere using any device and different access networks. It is anticipated that multimedia services will change the way we operate and interact with the world by developing numerous interesting multimedia applications. Interactive multimedia computing in ubiquitous environment poses many research challenges. One of the challenges is processing overhead in a service environment [2-7]. Therefore, novel techniques, architectures, algorithms, experiences regarding multimedia applications, and industry services should be considered, including the following fundamental issues.

- Interactive multimedia computing for convergence

- Multimedia and content protection

- Audio, video, image processing

- Digital signal processing for cluster

- Multimedia communications and networking

- Information system for interactive multimedia computing

\author{
R. Boutaba $(\bowtie)$ \\ School of Computer Science, University of Waterloo, \\ Waterloo, Canada \\ e-mail: rboutaba@uwaterloo.ca \\ K.-Y. Chung \\ Department of Computer Information Engineering, \\ Sangji University, Wonju-si, Republic of Korea \\ e-mail: kyungyong.chung@gmail.com

\section{Gen} \\ Fuzzy Logic Systems Institute, National Tsing Hua University, \\ Hsinchu City, Taiwan, Republic of China \\ e-mail: gen@flsi.or.jp
}

- Digital management for industry

- Interactive multimedia computer systems and appliances

- Intelligent iiformation extraction algorithm

- Multimedia indexing, searching, and visualization

- Interactive multimedia computing systems and database

This theme issue has become one of the hottest topics in interactive multimedia computing for industry. We believe that theme issue will have a high citation in the areas of cluster computing.

The paper, by Kwon et al., presents a method for monitoring in real time integrated environment and growth data and the energy consumption of the devices in a fully artificial plant factory, and to design and implement a plant factory integration management system that actively controls devices based on these data. The next paper, by Noh et al., has introduce a framework for virtual cluster system called vcluster which is capable of utilizing computing resources from heterogeneous clouds and provides a uniform view in computing resource management. They have introduced vcluster which is a framework to build a virtual cluster system in different types of cloud systems. The fundamental design approach of vcluster is agnostic on its underlying cloud systems and batch systems. The next paper, authored by $\mathrm{Oh}$ et al., presents an advanced taxi movement model in order to make the WDM more realistic. A taxi travels via the shortest path to a destination when there is a customer; otherwise, it moves at fast speeds randomly. Such movement can generate various communication situations and packet transmissions in a DTN. Therefore, the previous WDM was analyzed and a more proper design suggested.

The paper, authored by Lee et al., presents an experimental method for validating a tangible walking game that can be potentially applied to other serious games. A cognitive 
evaluation procedure has been developed showing how to exploit simple empirical testing to validate the effectiveness of the proposed tangible game through the cognitive aspects of information processing. This research was motivated by looking for a method of how the effect of a serious game can be verified. The next paper, authored by Jung et al., presents mining-based associative image filtering using the harmonic mean, which is based on the association between preferred image data. By adopting the preference mean regarding preferred images, this method overcomes the weakness of complicated calculation procedures. To evaluate the filtering method proposed herein, they used MAE to highlight gaps between the results of the proposed method and actual preferences. Utilizing MAE, this research found that the proposed method is $12 \%$ more accurate on average than other methods.

The paper, authored by Kim et al., presents a new non$\mathrm{PC} / \mathrm{SC}$ based mini WiMAX Connection Manager (non$\mathrm{PC} / \mathrm{SC} \mathrm{m}-\mathrm{WCM}$ ) that can be applied to small hardware system or architecture with micro controller unit. Thereby, they have proposed a non-PC/SC based mini WiMAX connection manager that can be applied to lightweight hardware systems with micro controller unit, and shown that it actually has no performance degradations compared to the legacy WCMs. The next paper, by Kwon et al., presents the linked data structure of community research and development information service, which is the R\&D information service adopted by the EU, and then suggest a case for utilizing the linked data format and design model for implementing the semantic web to effectively analyze the global national $R \& D$ trends by using a case where the RDF Triple data has been semantically mashed up from NTIS and NDSL.

The paper, by Lee et al., presents a bi-level modeling approach that captures hierarchical relationships between shippers and carriers in maritime freight transportation networks. Ocean carriers, land carriers, and port terminal operators provide transportation services at different parts of the multimodal network. The paper focuses on the behavior of shippers containing transportation service information within a spatial price equilibrium. A numerical example is used to demonstrate the validity of the developed model. The next paper, by Jeong et al., presents a model to convert a public institution financial reporting system into an XBRL-based online system in accordance with adopted Korean international financial reporting standards. Financial reporting systems before the adaptation have found themselves affected by many input errors in the process of manual collection of Excel-based data and combining.

The paper, authored by Han et al., presents a method for developing an intelligent recommendation system for automotive parts assembly. The proposed system displays detailed information and a list of components that the user wants through the database using ontology. It is possible to identify the otherwise undiscovered problems in the vehi- cle using the recommendation system and ensure stability when the user operates the vehicle. The next paper, authored by Choi et al. presents converged culture technology such as 3D-based CG, interactions, and network technology to create a storyboard writing tool. This tool can effectively produce pre-visualizations for different video types, including films. It can simulate videos and distribute digitally. This research allows for elaborate preparation. Effective pre-visualization and changes in the immediate field situation enable higher completeness of videos through cooperation between staff members. The next paper, authored by Lee et al., presents the interactions and relationships of different types of carriers in the oligopolistic and competitive shipping market, which is the current market environment for maritime freight transportation networks. Port terminal operators are especially regarded as a special type of carrier based on their behavior in making pricing and routing decisions. The paper contributes to the body of the current multimodal freight network problem literature.

The paper, authored by Kim et al., presents the effects of individual and social factors on a neutral outcome variable and the continuance of online gaming. In order to achieve this objective, they first investigated the possibility of replacing existing outcome variables, addiction or flow, with the continuance of online gaming. Second, they looked into the role of social interactions in online gaming; social interactions that occur in online games could strengthen the existing offlinerelationship or help gamers make new relationships. The next paper, by Kang et al., presents a cost function analysis to evaluate static task assignments as well as a heuristic algorithm for solving the transformed problem explicitly describing the tradeoff between goals. The simulation results showed that this approach outperforms the existing representative approach for a range of tasks and processing systems.

The paper, by Jung et al., presents a mobile healthcare application that can be used to check PHR-type EMR data of hospitals and managing diabetes. This application provides services for diabetes management based on categories of diabetes management, weight management, cardio cerebrovascular risk evaluation, stress and depression evaluation, exercise management, settings, and information. The next paper, by Kim et al., presents a fuzzy rule-based adaptive coronary heart disease prediction support model, which gives content recommendation to coronary heart disease patients. The proposed model uses a mining technique validated by medical experts to provide recommendations. The proposed method consists of three parts for heart disease risk prediction. This paper showed the importance of linking medical expert knowledge with data mining techniques, and it overcomes uncertainty in the prediction model using knowledge from medical experts to prevent coronary heart disease. The next paper, authored by $\mathrm{Oh}$ et al., presents an advanced taxi movement model in order to make the WDM more realis- 
tic. A taxi travels via the shortest path to a destination when there is a customer; otherwise, it moves at fast speeds randomly. Such movement can generate various communication situations and packet transmissions in a DTN. Therefore, the previous WDM was analyzed and a more proper design suggested.

The paper, by $\mathrm{Oh}$ et al., presents features a technique for extracting only the selected target voice from the input sound, which is a mixture of voices and noises. The feature for selective speech extraction composes a correlation map of auditory elements by using similarity between channels and continuity of time, and it extracts speech features by using a non-parametric correlation coefficient. This proposed model was validated by showing that the average distortion of separation of the technique decreased by 0.8630 $\mathrm{dB}$. The nest paper, by Park et al., has proposed a computational model for automatic acquisition of lexical knowledge based on the principles of human language information processing. The proposed model assumes a hybrid model for the human lexical representation including full-list and decomposition forms. The proposed method automatically acquires lexical entries and its grammatical knowledge by unsupervised learning techniques.

This fine collection of papers was achieved by fruitful collaborations. We gratefully acknowledge and express heartfelt thanks to all the authors for their worthy contribution to theme issue. We also would like to thank all the members of the ICCT Program Committee and anonymous reviewers for their help in identifying the novel papers and for their careful reading of earlier drafts in order to select 18 high quality papers out of 51 submitted papers, $35 \%$ acceptance rate. Furthermore, we would like to thank Professor Salim Hariri, editor-in-chief of the international research journals Cluster Computing, for his valuable remarks and his undeterred help throughout the publication process of theme issue.

\section{References}

1. Jung, H., Chung, K.Y., Lee, Y.H.: Decision supporting method for chronic disease patients based on mining frequent pattern. Multimedia Tools Appl. (2013). doi:10.1007/s11042-013-1730-3

2. Weiser, M.: Computer for the Twenty-First Century, pp. 94-104. Scientific American, New York (1991)

3. Chung, K.Y.: Recent trends on convergence and ubiquitous computing. Pers. Ubiquitous Comput. (2013). doi:10.1007/ s00779-013-0743-2

4. Jung, E.Y., Kim, J.H., Chung, K.Y., Park, D.K.: Home health gateway based healthcare services through U-health platform. Wirel. Pers. Commun. 73(2), 207-218 (2013)

5. Kim, S.H., Chung, K.Y.: Medical information service system based on human 3D anatomical model. Multimedia Tools Appl. (2013). doi:10.1007/s11042-013-1584-8
6. Lee, J.E., Chung, K.Y., Lee, K.D., Gen, M.: A multi-objective hybrid genetic algorithm to minimize the total cost and delivery tardiness in a reverse logistics. Multimedia Tools Appl. (2013). doi:10.1007/ s11042-013-1594-6

7. Gen, M., Lin, L.: Multiobjective evolutionary algorithm for manufacturing scheduling problems: state-of-the-art survey", pp. 18, J. Intell. Manuf. (2013). doi:10.1007/s10845-013-0804-4

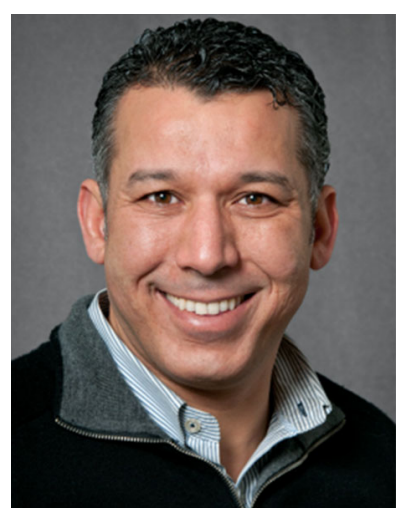

Raouf Boutaba is a Professor of Computer Science at the University of Waterloo and a David Cheriton Faculty Fellow in Canada. He is the founding Editor in Chief of the IEEE Transactions on Network and Service Management (2007-2010), and on the editorial board of several other journals. He served as the general or technical program chair for a number of international conferences. His research interests are in the areas of network and service management. He has published extensively in these areas and received several journal and conference Best Paper Awards such as the IEEE 2008 Fred W. Ellersick Prize Paper Award. He also received several other recognitions such as the Premier's Research Excellence Award, Industry research excellence Awards, fellowships of the Faculty of Mathematics, of the David R. Cheriton School of Computer Science and outstanding performance awards at the University of Waterloo. He has also received the IEEE Communications Society Hal Sobol Award and the IFIP Silver Core in 2007, the IEEE Communications Society Joe LociCero and the Dan Stokesbury awards in 2009, and the IEEE Communications Society Salah Aidarous award in 2012. He served as a distinguished lecturer for the IEEE Computer and Communications Societies. He is fellow of the IEEE and fellow of the Engineering Institute of Canada.

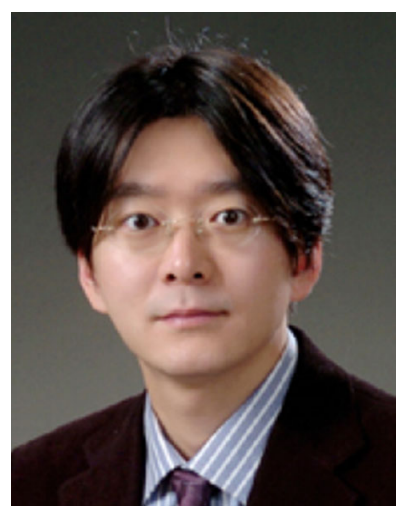

Kyung-Yong Chung is a professor of Computer Information Engineering Department at Sangji University, Korea. He has worked for Software Technology Leading Department, Korea IT Industry Promotion Agency (KIPA) in 2005. He received B.S., M.S., and Ph.D. degrees in Computer and Information Engineering from Inha University in 2000, 2002, and 2005, respectively. His research topics are Data Mining, Telemedicine, Knowledge based Decision Support System, Intelligent System, Convergence, HCI, and Recommendation. He serves as General Co-Chair of International Conference on Information Science and Application 2013, General Co-Chair of 2th International Conference IT Convergence and Security 2012, Executive Editing Director, Steering Committees of International Conference on Convergence Technology (ICCT). Also, he is editorial board members of several International Journals. 


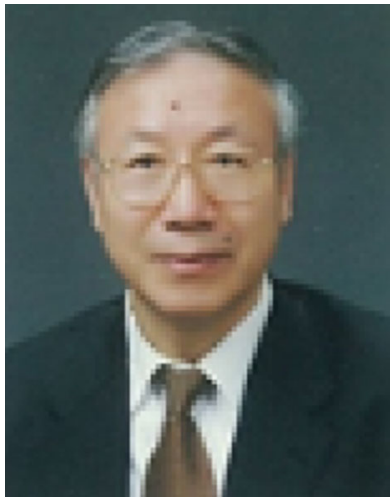

Mitsuo Gen is a senior research scientist at the Fuzzy Logic Systems Institute, Iizuka, Japan a visiting professor at National Tsing Hua University, Taiwan and a professor emeritus at Ashikaga Institute of Technology, Ashikaga, Japan. He graduated a $\mathrm{BE}, \mathrm{ME}$ and $\mathrm{PhD}$ in Electronic Engineering from Kogakuin University, Tokyo in 1969, 1971 and 1975 respectively and a $\mathrm{PhD}$ in Informatics from Kyoto University in 2006. $\mathrm{He}$ was a faculty at Ashikaga Institute of Technology, Ashikaga and a professor at graduate school of information, production \& systems, Waseda University, Kitakyushu, Japan. He was a visiting professor at IE\&OR Dept., University of
California, Berkeley in 1999-2000, IE Dept., Texas A\&M University, College Station in 2000 in USA, and a Hanyang chair professor at Hanyang University, Korea. His research interest includes Evolutionary Algorithms, Manufacturing Scheduling, Logistics Network and Decision Making. He is a coauthor for five books such as Introduction to Evolutionary Algorithms in 2010, Network Models and Optimization: Multiobjective Genetic Algorithm Approach in 2008 from Springer and others. He is one of Area Editors of Computers \& Industrial Engineering, Editorial Board members of several international journals. 